
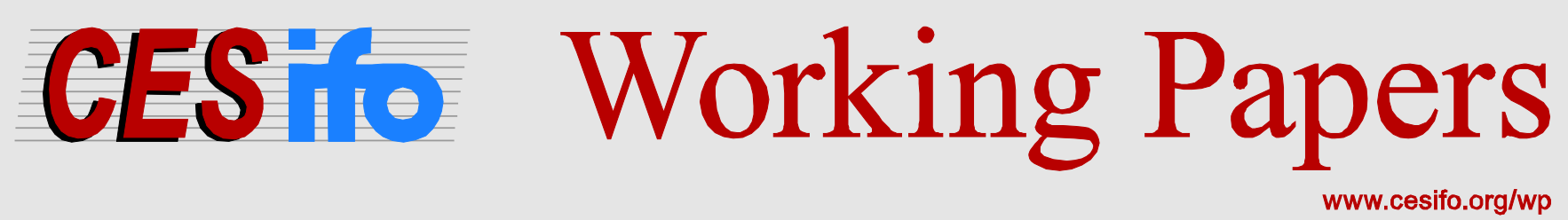

\title{
International Student Migration: A Partial Identification Analysis
}

\author{
Romuald Méango
}

\section{CESIFO WORKING PAPER NO. 4677 \\ CATEgORY 8: TRADE POLICY \\ FEBRUARY 2014}

Presented at CESifo Area Conference on Global Economy, May 2013

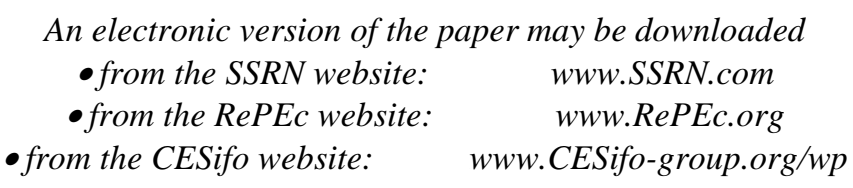




\title{
International Student Migration: A Partial Identification Analysis
}

\begin{abstract}
This paper studies the decision made by a family to invest in student migration. We propose an empirical structural decision model which reflects the importance of both the return to the investment and the budgetary constraint in the choice of the family. We circumvent the problem of endogeneity of the educational attainment by deriving sharp bounds and conduct inference for the parameters of interest. The data are collected on students from Cameroon, using a new snowball sampling procedure, which allow the inclusion of both migrants and non-migrants in the sample. We propose bias corrected estimators for this procedure. We study the characteristics of potential candidates to migration that increase or decrease their probability to migrate, accounting for a potential helper in the diaspora. Among the interesting results we find that a choice to complete a Master's degree doubles the odds of migration, there is little evidence of gender preference, students migrants are positively selected on their previous academic results.
\end{abstract}

JEL-Code: C130, C250, D850, I250, J610.

Keywords: student migration, network sampling, incomplete structural models, partial identification.

Romuald Méango
Ifo Institute - Leibniz Institute for Economic Research
at the University of Munich
Poschingerstr. 5
Germany-81679 Munich
meango@ifo.de

Date: February 8, 2014. ifo Institut, Munich. Financial support from SSHRC Grant 410-2010242 and Bourse d'étude de Fin d'étude Doctorale of Université de Montréal are gratefully acknowledged. The survey implemenation has been reviewed and approved by the Comite d'éthique de l'Université de Montréal. This research was conducted in part while I was visiting UCL/CeMMAP. I thank Marc Henry for his availability and insightful comments, Silvia Gonçalves, Onur Özgür, Lars Ehlers, Adam Rosen, Martin Weidner and Ismael Mourifié for helpful discussions, Joël Cornuz for invaluable help with the online application. I also thank participants to the 2013 CESifo conference on Global Economy, CeMMAP/WIP and UCL SWIP seminar for helpful comments, and participants to the survey. All remaining errors are mine. 


\section{INTRODUCTION}

As the number of students choosing to study abroad has considerably increased since 1970's, the interest of the economic literature in this specific form of migration has also grown in the recent years. There is at least to features that make the specialization to this topic relevant. First, student migration may be seen as a route to permanent emigration. As pointed out by Rosenzweig (2008), unlike for many visas, there are no country ceilings or kinship requirements for student visas. In addition, the probability that a foreign-trained student will remain in the host country is higher than the overall emigration probability for a domestically schooled student. Finally, universities are often of better quality and for comparable skills levels, remuneration are higher in high-income countries. Entering the host country as a student appears therefore as a dominant strategy for prospecting migrants. Second, due to higher costs of living and potentially higher fees in foreign countries, the budget constraint plays an important role. The family (and not only the student) resources matter. According to data released by the Institute of International Education (IE) in 2006, the primary source of funding is "personal and family" for about 64 percent of foreign students.

However, mainly because of data constraints, the focus of the literature has been largely directed to macroeconomic analyzes of determinants of student mobility, Batista, Lacuesta, and Vicente (2012) (BLV, hereafter) being one notable exception. The recent contributions from Rosenzweig, Irwin, and Williamson (2006), Rosenzweig (2008), Beine, Noel, and Ragot (2012) and Perkins and Neumayer (2011) among others build on the findings of the more established literature on international migration to study determinants in origin and/or destination countries that encourage, attract or retain student migrants, the so-called push and pull factors. What so far received less attention are the determinants of the choice of location of tertiary studies from a microeconomic perspective. Most importantly, how influential are the choice of final level of education, the characteristic of the candidate to migration, as well as family's socio-economic characteristics?

As stated earlier, the nuclear family is highly involved in migration choices and there characteristics should be included in any analysis of the decision process. Moreover, as documented by survey results and the literature of student migration (see for example Perkins and Neumayer (2011)), migrants might receive some support from the diaspora in their destination country, e.g a distant relative of the student's family living in the destination country provides some help by hosting the migrant, providing food and accomodation. This effect, known in the migration literature as a network effect, provides some implicit capital to the family. The characteristics of this helper should therefore be relevant determinants within our analysis. 
But engaging in this study, one faces major challenges, the main of them being the data limitations. Most existing empirical studies are based on country-level samples which fail to reflect the heterogeneity within a specific community. Conversely, as the population of interest is often scattered around the globe, micro-level samples are easily in danger of missing part of the population of interest. Furthermore, the pervasive problem of endogeneity renders the econometric analysis difficult. In that strong structral assumption are required for identification and estimation of the structural parameters, the robustness of the methods in use so far (in BLV for example) is debatable. These challenges explain the scarcity of the literature on the microeconomic determinants of international student migration.

The objective of this paper is to provide a rigorous framework to study the investment decision made by the family of the candidate to emigration, while addressing the above concerns. Throughout the paper, we work with a structural discrete choice model of private investment of the family in the student migration. Reminescent of the human capital litterature, our framework assume education of a child to be an investment of the family unit which seeks to maximize it lifetime earning. The family decides of the location of tertiary study of the sudent; however, this choice is contingent on the choice of final education level (hence the problem of endogeneity). Migration occurs when the expected return of the investment is positive. Our model disentangles the liquidity constraints effects from the expected return of the investment. We are interested in the determinants of the return component for the family, as well as the deterring effect of the financial constraint component. The novelty of our approach is that, albeit a monotonicity condition, we do not impose any restriction on the relationship of the educational attainment and migration, neither do we rely on a identifying instrument.

The contributions to the literature on the topic are three-fold: first, we take advantage of recent developments in network sampling to construct a novel dataset on a population of students from Cameroon, migrants and non-migrants. Second, we show that our structural model of investment in student migration offers itself quite nicely to an inference procedure in an incomplete (partially identified) model framework. This has two major advantages: frist, the structural assumptions are fairly parsimonious because mainly driven by the economic analysis and not impose to solve technical shortcomings. Furthermore, we do not appeal to the use of a identifying instrument, hence, circumventing the problems of validity and relevance. Following the proposal in Chesher, Rosen, and Smolinski (2011), we use sharp bounds for our discrete choice models, with endogenous right-hand side regressor. These bounds translate in moment inequalities and a large literature has developed in this field. A challenge though remains the computational burden of these methods, which is often increased when considering models with relatively high number of covariates. Henry, Méango, and 
Queyranne (2011) (HMQ hereafter) proposes a combinatorial approach to solve the above problem and their method is the best suited to inference in our framework. As a final contribution of this paper, we propose a methodology to preestimate a number of parameters of interest, decreasing significantly the computational requirement. This step appears crucial, since the sample size required to achieve informative inference, grows rapidly with the number of covariates.

For the purpose of the study, a survey has been conducted on the population of Cameroonian, aged 18 or more, having completed secondary school by obtaining the "Baccalauréat" ${ }^{1}$. We gathered information on 418 respondents. Our survey data show that close to three quarters of migrant rely on themselves or on family capital to finance their study abroad. More than half of the respondants reports the existence of a potential or effective helper in the migration process. The "typical" helper is a male, an uncle or a brother who have a university degree. Unsurprinsingly, families where at least one of the parent have higher level of education, and families who possess higher physical capital, have less difficulty to meet the budget constraint for migration. Concerning determinants of the return of investment in migration, we find that a higher choice of educational attainment along with better candidate's results during secondary school significantly increase returns to migration. This finding suggests a positive selection of migrants. Interestingly, first born-child has lower probability of migration than the subsequent children, while the gender does not seem to affect the probability of migration.

This paper is mainly related to three strands of literature, the Network Sampling literature, the student-mobility and the more general migration literature and the partial identification literature. With the recent expansion of social networking services and advances in computational capabilities, a renewed interest has grown for methods of data collection over networks. The procedure we propose has been inspired by the Respondant-Driven Sampling methodology proposed by Heckathorn (1997, 2007) and Wejnert and Heckathorn (2008). To estimate inclusion probabilities and correct for oversampling of some population members, we use estimators first applied by Thompson (2006) in the context of network sampled data.

The incentives for international migration of skilled individuals have been extensively studied by a number of early key contributions for which the surveys by Borjas $(1989,1994)$ serve as good references. Rosenzweig (2008) studies out-migration of (Asian) students. One of his contributions is to distinguish the effects of the return of out-migration and budgetary constraint on a candidate decision. Both appear important in the final decision of migration. Beine, Noel, and Ragot (2012) and Perkins and Neumayer (2011) study the determinants of migration in multi-origin multi-destination

\footnotetext{
${ }^{1}$ Similar to the french educational system, "Baccalauréat" is a compulsory state exam for completion of secondary school.
} 
framework. Among interesting results, they find a strong effect of the number of migrants in the destination country on the probability of migration.

Micro-level studies of the question of the incentives of international student migration are still rare. BLV studies the case of Cape Verde by taking advantage of a specifically tailored survey on households in the country. They are interested in testing the brain gain effect, which in their framework, amounts to testing for a significant linear correlation between the own future probability of migration and the schooling decisions. To achieve identification, their econometric model relies on a distributional assumption on the joint behavior of the latent variable and on some exclusion restrictions. Additional, though not exhaustive, reference on international student migration includes Dreher and Poutvaara (2011), Bessey (2011), Brezis and Soueri (2011), Thissen and Ederveen (2006), Van Bouwel and Veugelers (2009).

Our work is also related to an increasing literature on partially identified models, following the seminal works of Manski (1993) and Jovanovic (1989). Closely related to our framework, the papers of Beresteanu, Molchanov, and Molinari (2011), Galichon and Henry (2011) and Chesher, Rosen, and Smolinski (2011), which relax the requirement for point identification in structural models and derive sharp bounds on the parameters of these models. The first two explore the case of strategic games in complete information, while the last one is concerned with instrumental variable model of discrete choice. As the bounds translate into moment inequalities, our inference procedure is related to a large literature which has developed on inference in moment inequality models since the seminal contribution of Chernozhukov, Hong, and Tamer (2007). A major challenge is the computational burden of these methods, here aggravated by the relatively high number of covariates.

In Section 2, we present a family investment decision model in a human capital framework. Section 3 is devoted to the inference procedure. The data collection procedure is presented in Section 4. Finally, we gather the results of the inference on the parameters of the structural model in Section 5 , before we conclude. Proofs are collected in an online appendix ${ }^{2}$

\section{A structural Model of Private Investment in Student Migration}

Student migration displays two important characteristics. First, the choice of location of tertiary education is the result of an arbitrage between the schooling and employment opportunities available in the origin country and in the host country. Second, the amount of capital that the family can

\footnotetext{
${ }^{2}$ Available at the following address: https://dl.dropboxusercontent.com/u/2917549/migcam_supplementary_material.pdf.
} 
invest in the process is key to the migration process. The model we present mirrors these two essential characteristics.

Following Beine, Noel, and Ragot (2012) (see also Brezis and Soueri (2011)), we consider a framework based on the human capital literature, where "Education is considered as an investment in future earnings and employment for rationale [families] who seek to maximize the lifetime earnings." The decision of a student migration is then between (1) obtaining further education in a foreign country or (2) studying or starting professional activities at home. Of course, this decision does not preclude further international mobility when education is completed, although the above choice affects significantly the probability of later migration - student migrants have better opportunities on the labor market of their host country (some evidences in Rosenzweig (2008)). Of equal importance, student migrants might return to their origin country to work. Our explicit assumption is that the choice of location of tertiary education and the choice of location of work are taken sequentially. Note that the benefits discussed here are not only pecuniary. For example, the utility enjoyed by having a child entering the marriage market of the host country, or the disutility of having a child living to a greater distance (e.g for provision of health care to elderly parents).

To be more specific, the evaluation of benefits depend on two main components: one that depends on the characteristics of the child. Of particular interest for us, his/her final educational attainment. The unobserved innate ability of the child also influences how likely he is to adjust to life in a foreign culture or how much he prefers staying in his country ${ }^{3}$.

The other important part, on which these benefits depend on, is the wealth of the family. Indeed, obtaining tertiary education in a foreign country which has often higher income per-capita entails significant costs. Education costs, travel costs and living costs. The family could possess some implicit capital that will lower the financial input required for the student migration investment. In particular, if a relative lives in the destination country. This relative could provide material and financial support. Family characteristics will therefore influence the amount of money that the familly needs to borrow for the student migration investment, if any. The availability of a scholarship for the student might also significantly relax the budget constraint of the family.

The families form myopic expectations on the return on studying abroad or staying home, observing skill prices and probabilities of later settlement in a foreign country. The family compares returns on both alternatives, and invest in migration when the migration option yields the largest expected benefits. We introduce more formal notation in the following.

\footnotetext{
${ }^{3}$ This component could be extend to specific preferences of the family.
} 
Consider a family indexed by $i$. Here we think of an extended family (parents, child and some relative of the family who might provide some support) with one member (a child) who is a potential migrant. The family possesses a capital $K_{i}^{0}$ (explicit or implicit, as discussed above). The child has observable characteristics $X_{i}$ (e.g. education, gender and previous academic result of the child) and characteristics $\varepsilon_{i}$, known to all family members but unobservable for the econometrician (innate ability of the child, cultural factors influencing evaluation of the migration alternative,etc.). When the child complete secondary school, the family has the opportunity of making an investment of level $I$, known and fixed across families, by financing further education of the child in a foreign country. The reader should understand $I$ as the minimum financial input enabling student migration to the country of destination. It includes travel, education and living expenses. We call $I_{f}\left(E d_{i}, X_{i}, \varepsilon_{i}, \theta\right)$ the gross revenue from the investment and $r\left(E d_{i}, X_{i}, \varepsilon_{i}, \theta\right)$, its return, which both depend on the observable and unobserved characteristics of the family. Note that the two variables are linked by the following equation :

$$
r\left(E d_{i}, X_{i}, \varepsilon_{i}, \theta\right)=\frac{I_{f}\left(E d_{i}, X_{i}, \varepsilon_{i}, \theta\right)}{I}-1
$$

$\theta$ are here the parameters of interest. $E d_{i}$ is the final education level. The choice of education will affect the salary the agent expect after graduation but also the probability of subsequent migration ${ }^{4}$. The endogeneity problem will arise primarily from the potential correlation between the unobservable variable $\varepsilon_{i}$ and the choice of final educational attainment. If $K_{i}^{0}<I$, the family must borrow capital to be able to make the investment. Denote $r_{0}$ the interest rate for borrowing. In the following, we will reason in term of return or interest rate.

As mentioned above, the student has the alternative to remain in his origin country, either to obtain further education or to work. Again, later on, the individual decides to migrate or not and his/her prospect of migration enters the valuation of this given alternative. We will assume that this alternative, along with other potential investment alternatives in the origin country, yield an interest rate on the family capital $K_{i}^{0}$, that we will denote $r_{1}$. By assuming $r_{1}$ to be constant, we implicitly assume that the return on the investment of the family capital in the origin country, is not (or only mildly) affected by the choice of final education. This would be the case if the return to skills is low in Cameroon and the probability of work migration when schooling has been completed in the origin country is low and not very sensitive to the choice of education. As this seems to be case in the country where we conduct our empirical application, for the level of education we consider (Masters), we do not expect our result to be very sensitive to this hypothesis (see IOM

\footnotetext{
${ }^{4}$ Dreher and Poutvaara (2011) points out that " host countries are interested in educating foreign students, partly to attract human capital benefiting the domestic economy". Educational attainment and probability of settlement in the host country increase together for a migrant student.
} 
report, 2010). As the reader might expect, education in many developing country is pretty cheap. However, it yields low expected returns, we impose $r_{1} \leq r_{0}{ }^{5}$.

The family compares investments with respect to their expected return. Knowing the interest rates above, the family chooses the investment alternative to maximize its expected profit that we will denote $\Pi_{i} \equiv \Pi_{i}\left(E d_{i}, X_{i}, K_{i}^{0}, \varepsilon_{i} ; r_{0}, \theta\right)$ (to simplify notation, we drop the subscript $i$ in the subsequent development). Choosing to remain in the origin country yields an expected profit of :

$$
\Pi=r_{1} K^{0}
$$

While the investment student in migration, along with a choice of final education level of $E d$ gives:

$$
\Pi=r(E d, X, \varepsilon ; \theta) I+\left(K^{0}-I\right)\left(r_{1} 1\left\{K^{0}-I>0\right\}+r_{0} 1\left\{K^{0}-I<0\right\}\right)
$$

with $1 A=1$ if $\mathrm{A}$ is true, and 0 if not. The net profit of the student migration for the family can then be written:

$$
\tilde{\Pi}=\left(r(E d, X, \varepsilon ; \theta)-r_{1}\right) I+\left(r_{0}-r_{1}\right) \min \left(K^{0}-I, 0\right)
$$

Note that $\left(r_{0}-r_{1}\right)$ measures the effect of the budget on the net profit of student migration. If $r_{0}=r_{1}$, the family must simply compare its return from the student migration investment to the return of alternative investment in the origin country. All that matters in the decision is the additional return of investment and the budget constraint plays no role. We can simplify Eq. (2.1) to

$$
\frac{\tilde{\Pi}}{I}=r(E d, X, \varepsilon ; \theta)+r_{0} \min \left(\frac{K^{0}}{I}-1,0\right)
$$

and $r($.$) and r_{0}$ can be reinterpreted "net of the interest rate $r_{1}$ ". We assume the following separable formulation of return function:

$$
r(E d, X, \varepsilon ; \theta)=\tilde{r}(E d, X ; \theta)-\varepsilon
$$

and define finally:

$$
\tilde{\pi}\left(E d, X, \frac{K^{0}}{I} ; r_{0}, \theta\right) \equiv \tilde{r}(E d, X ; \theta)+r_{0} \min \left(\frac{K^{0}}{I}-1,0\right) .
$$

The investment decision being denoted $Y \in\{0,1\}$, where 1 means that the family chooses student migration, is then characterized in the following way:

$$
Y=1\left\{\tilde{\pi}\left(E d, X, \frac{K^{0}}{I} ; r_{0}, \theta\right)-\varepsilon \geq 0\right\}
$$

\footnotetext{
${ }^{5}$ For reference, the interest rate spread (the difference between the lending and the borrowing rate) in Cameroon is about 0.2 for "tontines" which are very popular among Cameroonian households for financing even very large investments (Nemb and Jumbo (2011))
} 
We will assume later in Section 5 a linear return in the characteristics (see Eq. 5.1). It is assumed that the variable $\varepsilon$ follows a logistic distribution of variance normalized to 1 . In an abuse of notation in the following and when the context is clear enough to allow it, we will refer to the structural parameters of the function $\tilde{\pi}$ as $\theta$, while meaning the pair $\left(r_{0}, \theta\right)$. The parameter $r_{0}$ will receive special attention in Section 3.4.

2.1. Treatment of endogeneity of the educational attainment. Among the observable characteristics influencing the migration decision, the schooling attainment of the candidate must be seen as an endogeneous variable. The nature of the potential endogeneity is here two-fold. First, the innate ability remains an omitted variable correlated with the educational attainment. This problem is well known in the labor literature. For example, the signaling model in Spence (1973), agents with high innate ability are assumed to find school less difficult and obtain higher education as a signal of their high ability (see Willis (1985) for survey). Second, as recognize in the migration literature, education choice and migration decision are often simultaneously decided at household level (Hanson and Woodruff (2003), McKenzie and Rapoport (2007)). A change in educational attainment affects the decision to migrate, which affects the working migration probabilities, impacting in turn the return of student migration, and so forth. It is easy in fact to agree with the claim that more education decreases the cost of migration procedures and, therefore, makes it more likely to migrate. Indeed, Docquier and Marfouk (2006) find that emigration propensities are five to ten times higher for workers with more than twelve years of education than for workers with less than twelve years of education. In the other direction, the prospect of migration could be the incentive to acquire more education since it ensures higher returns abroad. Rosenzweig (2008), for example, points out that the choice of location of tertiary education significantly affects the probability that a person can emigrate permanently.

The common methodology to deal with these two issues would amount in our framework to specify further the relationship between the migration and schooling decision, in the form of a simultaneous probability model. Identification and estimation methodology then rely on a structural assumption about the error terms (bivariate normal distribution), as well as strong (identifying) exclusion restriction (see for example Mallar (1977)). Namely, we need variables that affect the choice of final education level and are known at the time the migration decision is made, but which do not directly affect the migration decision. This methodology is used by BLV, albeit the fact that they rather treat the problem of endogeneity of the migration variable in the equation of the schooling choice. Our main critics to this methodology are two-fold: first, the imposed structural assumption on the error terms are made for technical reason rather than sound economic intuition. Second, the exclusion restriction should be teamed with conditions on the support of the excluded variable 
to obtain point identification. The argument is similar to the one in Ciliberto and Tamer (2009) (Theorem 2): identification of parameters occur through "independent variation in one regressor while driving another to take extreme values on its support (identification at infinity)". Altogether, these assumptions are arguably very strong.

The strength of the methodology presented in this paper resides in that we need not to model any further the relationship between Educational attainment $(E d)$ and migration $(Y)$ to conduct inference, albeit a condition on the ordering of returns on migration for different level of education, all other variables being controlled. In particular, the inference procedure does not require the existence of a valid instrument. The model rests therefore upon fewer structural assumption. It comes at the cost of losing identification of parameters. Indeed, allowing for endogenous explanatory variable in discrete choice models might hinder point identification of parameter of interests. Early treatments of this problem can be found in Manski (1993).

In this paper, we pursue the avenue of set identification rather than point identification. Recent contributions made by Beresteanu, Molchanov, and Molinari (2011), Galichon and Henry (2011) (GH, hereafter) and Chesher, Rosen, and Smolinski (2011) (CRS, hereafter) show that, without additional hypothesis to the model, bounds can be derived on the structural parameters of an IVdiscrete choice model. We present these results in the context of our model and show how to conduct inference, following the proposal of HMQ.

\section{INFERENCE PROCEDURE}

In the model described above, we are first interested in the determinants of migration. We build on the work of GH, CRS and HMQ to conduct inference for the parameters of the structural equation (2.2). A confidence region is derived by using sharp bounds on the structural parameters. However, because some data are incompletely observed, further complications are added to the derivation of the sharp bounds, independently of the inference procedure. In the following, we present sharp bounds on the structural parameters of the model in its simplest form. On this simple model, we show how to conduct inference. Section 3.3 then deals with the complications mentioned above.

3.1. Sharp bounds. Suppose that we observe $Y \in \mathcal{Y}, E d \in \mathcal{E}$ and $X \in \mathcal{X}$ on a family $i$, as defined by the previous section. We suppose in this section that we can also observe $V \equiv \frac{K_{0}}{I}-1$, the fraction of the investment that the family needs to borrow. Note that $V$ is a random variable distributed on the real line. We denote the vector $W \equiv(X, V)$. The unobservable latent variable is $\varepsilon$, distributed on the real line. It summarizes the decision shifters that are known to the family but unknown to the econometrician. Our interest is primarly in inference on $\theta$, a finite dimensional parameter which 
characterizes the return on student migration of the family. With the above notation, the utility of the family can then be rewritten:

$$
\tilde{\pi}\left(E d, W ; r_{0}, \theta\right)-\varepsilon=r(E d, X ; \theta)+r_{0} \cdot \min (V, 0)-\varepsilon
$$

We assume that $X$ and $V$ are exogeneous in the sense that $\varepsilon$ and $W$ are stochastically independent. The above assumption implies in particular the innate ability of the child will not be influenced by the capital possessed by his family. Let $F_{E d, Y \mid W}^{0}$, the distribution of the $(E d, Y)$ given $W$, and $F_{\varepsilon ; \theta}$, the distribution of $\varepsilon$ given $W$ on $\mathbb{R}$. We will denote the respective densities accordingly $P_{0}(. \mid W)$ and $P_{\varepsilon}(. \mid W)$. As noted above, endogeneity of the random variable $E d$ might preclude point identification as several parameter values $\theta$ from the model could be consistent with the data. We define $\Theta_{I}$ this set of parameter. A model which singles $\Theta_{I}$ as the set of possible values for $\theta$ is said to be set identified. Point identification occurs when $\Theta_{I}$ is reduced to a singleton. A model is rejected by the data if $\Theta_{I}$ is the empty set.

We give here the sharp bounds induced on the structural parameters for a model with two alternatives of migration and two levels of education.

Theorem 1 (Sharp bounds). Consider the set of parameters $\theta$ for which we have positive return on student migration to education, i.e.

$$
\tilde{\pi}(1, w ; \theta)>\tilde{\pi}(0, w ; \theta)), \text { for all } w \text {. }
$$

A parameter $\theta$ belongs to the identified set, if and only if we have:

$$
\begin{aligned}
P_{0}(Y=1, E d=0 \mid W=w) & \leq F_{\varepsilon}(\tilde{\pi}(0, w ; \theta)), \\
P_{0}(Y=1, E d=1 \mid W=w) \leq & F_{\varepsilon}(\tilde{\pi}(1, w ; \theta)), \\
P_{0}(Y=0, E d=0 \mid W=w) \leq & 1-F_{\varepsilon}(\tilde{\pi}(0, w ; \theta)), \\
P_{0}(Y=0, E d=1 \mid W=w) \leq & 1-F_{\varepsilon}(\tilde{\pi}(1, w ; \theta)), \\
P_{0}(Y=1 \mid W=w) \leq & F_{\varepsilon}(\tilde{\pi}(1, w ; \theta)), \\
P_{0}(Y=0 \mid W=w) \leq & 1-F_{\varepsilon}(\tilde{\pi}(0, w ; \theta)), \\
& w \text { a.e. }
\end{aligned}
$$

Theorem 1 is a direct consequence of Propositon 1 in the online appendix. The terms in the left-hand side are derived from the Data Generating Process of the observable variables. Note that they do not include the parameter $\theta$. The terms in the right-hand side are derived from the cumulative distribution of the latent variable $\varepsilon$. To understand these inequalities, we can think 
of the observed outcome $(y, e)$ as part of a multiple equilibrium predicted by the model. For example, let $\theta_{0}$ be the true parameter. $(Y=0, E d=1)$ can only be observed if $\tilde{\pi}\left(0, w ; \theta_{0}\right) \leq \varepsilon$ (even after obtaining education $E d=1$, the return of migration are not large enough to make migration attractive). But if the latter is true, the model predicted also that $(Y=0, E d=0)$ was a possible outcome i.e, returns to migration are also too small for those who choose education $E d=0 . \quad\{(Y=0, E d=1) ;(Y=0, E d=0)\}$ can be understood as a multiple equilibrium prediction. In other words, we cannot observe $(Y=0, E d=1)$ more often than the model predicted $\{(Y=0, E d=1) ;(Y=0, E d=0)\}$. Hence, (3.6). Theorem 1 summarizes this fact: the probability that we observe a given outcome, cannot be greater than the probability that the model predicts at least one of all the multiple equilibria where this outcome is part of.

Under the condition (3.2), $\Theta_{I}$ is defined as the set of parameters for which (3.3) - (3.8) are true. These bounds which can easily be derived as necessary conditions, are also sufficient (see online Appendix), hence the term "sharp" bounds. Note that some of these inequalities are redundant, and only a subset of those is enough to characterize $\Theta_{I}$. We now turn to the inference procedure.

3.2. Inference on the structural parameters. Condition (3.2) is not a stochastic condition which will be assumed through out the rest of the paper. Here, we seek coverage of the identified set, $\Theta_{I}$ with a prescribed probability, $1-\alpha$. The idea of the procedure is to define a new set of inequalities which relaxes the bounds with a definite probability $1-\alpha$, so that a parameter satisfying (3.3) - (3.8) will satisfy this new set of inequalities with confidence level $1-\alpha$. The confidence region will simply be the collection of all those parameters satisfying the new set of inequalities. The relaxation occurs through the construction of a function $\underline{P}_{n}$, dominated by the probability distribution $P_{0}$. Suppose indeed that we can construct a function $\underline{P}_{n}$ such that for all $(y, e) \in\{0,1\} \times\{0,1\}$, and $w$ a.e.:

$$
\underline{P}_{n}(Y=y, E d=e \mid W=w) \leq P_{0}(Y=y, E d=e \mid W=w)
$$

with probability $1-\alpha$. A new set of inequalities (3.3') - (3.8') can be obtained by replacing $P_{0}$ by $\underline{P}_{n}$ in (3.3) - (3.8). Define now $\hat{\Theta}_{n}$ has the collection of parameters $\theta$ satisfying (3.3') - (3.8'). Theorem 1 in the online appendix shows that we achieve with $\hat{\Theta}_{n}$ a proper coverage of the identified set $\Theta_{I}$.

Construction of a functional satisfying (3.9) is proposed by HMQ through a procedure called "efficient combinatorial bootstrap" that runs in linear computing time. It involves bootstrapping the empirical process of the distribution $P_{0}(. \mid W)$, to retrieve the $(1-\alpha)$-quantile of this process, $c_{\alpha}(W)$. Then, the empirical distribution $\hat{P}_{0}(. \mid W)$ is decreased by $c_{\alpha}(W)$. This decreased quantity offers the desired functional $\underline{P}_{n}$. 
Although HMQ only covers the case of discrete variables, results from Chernozhukov, Lee, and Rosen (2009) (Section 4.1) makes it sensible to use the procedure in the parametric case.

3.3. Incomplete observation of Educational attainment. The respondent's educational attainment is not completely observed unless he/she has completed her study. In fact, $48 \%$ of the respondents have not. Information are available though on the level of education at the time of response, which we will denote it $\underline{E d}$. To complement this information for those respondants who had not yet completed there studies, a question in survey was relative to their highest expected educational attainment. this variable is then observed in our dataset and we will denote it $\overline{E d}$. Under the assumption that a person does not study more than the expected level, i.e. $\underline{E d} \leq E d \leq \overline{E d}$. We then have an interval $[\underline{E d} ; \overline{E d}]$ such that:

$$
\mathbb{P}(E d \in[\underline{E d} ; \overline{E d}])=1
$$

This information can be incorporated to the earlier framework as a censored variable problem. The non-redundant sharp bounds become:

$$
\begin{aligned}
& P_{0}(Y=1, \underline{E} d=0, \bar{E} d=0 \mid W=w) F_{\varepsilon}(\tilde{\pi}(0, w ; \theta)), \\
& P_{0}(Y=0, \underline{E} d=1, \bar{E} d=1 \mid W=w) \leq 1-F_{\varepsilon}(\tilde{\pi}(1, w ; \theta)), \\
& P_{0}(Y=1 \mid W=w) \leq F_{\varepsilon}(\tilde{\pi}(1, w ; \theta)), \\
& P_{0}(Y=0 \mid W=w) \leq 1-F_{\varepsilon}(\tilde{\pi}(0, w ; \theta)), \\
& w \text { a.e. }
\end{aligned}
$$

See Proof in the online appendix, section 1.

\subsection{First-step estimation of V: the proportion of the investment that the family needs}

to borrow. We assumed in Section 3.1 that the realization of the random variable $V$, the ratio of the capital of the family to the amount to invest, was observable for each family. As in many other studies, this information is absent from the dataset. The "natural" method to get around this problem, would be to add to the covariates, all observable variables which are known to influence the capital of the family, and conduct inference with this new set of covariates. This, however, by augmenting the dimensionality of $W$, increases the size of the data required to achieve informative inference and the computational burden. We use instead additional information provided by the survey to devise a two-step estimation in the same spirit as an Heckit estimation. This simplification will however be at the expense of informativeness of inference relative to the parameter measuring the effect of the budget constraint. 
The dataset allows to distinguish families who need to borrow from families who have sufficient funds to cover all the costs of the migration investment. In other words, we observe $1\{V<0\}$ instead of $V$. From this information and the observation of other socio-economic characteristics of the family, we construct a confidence region for the realization of $V$. Indeed, denote $L_{i}$, the observable socio-economic characteristics of the family $i$. We postulate for $V_{i}$ the following single index functional form:

\section{Assumption 1.}

$$
V_{i}=\beta L_{i}+u_{i} \text { where } u_{i} \text { follows } N\left(0, \sigma_{u}\right)
$$

where $u$ is stochastically independent of $\varepsilon$ given $(W, L)$.

See the variables in included in $L$ in Table 1 .

Since $1\{v<0\}$ is observed, the parameter of this model can be estimated through a probit estimation, under a scale normalization. One word of caution is therefore required here. If $\sigma_{u}$ were known, for a real $0<\alpha_{v}<1$, we would then have an interval $[\underline{v} ; \bar{v}]$ such that:

$$
\mathbb{P}(V \in[\underline{v} ; \bar{v}])=1-\alpha_{v}
$$

Our inference problem becomes one where the covariates are defined by an interval rather than a point. By an appeal to the Composition Theorem of Galichon and Henry (2006) (Theorem 1), we can redefine our identified set and propose a valid confidence region, following the same procedure as in Sections 3.1 and 3.2. However, $\sigma_{u}$ is a nuisance parameter here and the estimation procedure requires a scale normalization. As the reader might expect, the probit model under the standard normalization $\sigma_{u}=1$ gives us not a confidence interval for $V$, but for a variable $V_{\mid \sigma=1}$, related to the original variable by the relation:

$$
V_{\mid \sigma=1}=\sigma_{u}^{-1} V .
$$

The nuisance parameter $\sigma_{u}$ will affect inference on the parameter $r_{0}$. Indeed, using $V_{\mid \sigma=1}$ Equation (3.1) can be rewritten.

$$
\tilde{\pi}\left(E d, W ; r_{0}, \theta\right)-\varepsilon=r(E d, X ; \theta)+r_{0} \cdot \sigma_{u} \cdot \min \left(V_{\mid \sigma=1}, 0\right)-\varepsilon
$$

The bounds that we obtain will be informative for the parameter $r_{0} . \sigma_{u}$. However, since $\sigma_{u}$ remains unrestricted, the model will be uninformative on the parameter $r_{0}$

Note that, the inference methodology is in two steps, reminiscent of the two-steps of an Heckit estimation. Prior to the inference in our partially identified framework, we perform a first step estimation to overcome the computational burden induced by the high number of parameters. This first step estimation consists in a probit of some components of the parameter vector $\theta$. Without 
it, the partial identification inference would be computationally infeasible, since it would involve searching over a parameter grid with an unreasonable size. The price to pay is uninformativeness of our procedure on the parameter $r_{0}$. A mathematically rigorous treatment of the inference procedure in a general case is offered in the online appendix for interested readers.

\section{Database}

We begin this section by providing some facts on Cameroon, which we believe are relevant in understanding the background of the study. The main part of this section (Subsection 4.2) is devoted to the core of the snowball sampling methodology used for the online survey. We explicit the sampling design and show, under simplifying assumptions, how to estimate the population proportions by correcting for non-response and bias in the survey sampling design. Finally, in Section 4.3, we discuss the descriptive statistics drawn from the dataset.

4.1. Snapshot of Cameroon. Cameroon ${ }^{6}$ has an estimated population of 19.5 million in 2009, relatively young: an estimated $40.9 \%$ are under 15 . After a severe recession period from 1985 to 2000 , due to the fall in price of raw material exports, the nominal GDP returned to a steady growth from 2001, with an annual rate around 3\% from 2004 to 2009 . However, $30 \%$ of the population lives with less than $2 \$$ per day in 2007. The educational system in Cameroon is a mixture of British and French precedents. The typical curriculum consists of 6 years in primary school and 7 years in secondary school. Access to university is conditional on passing the state exam, "Baccalauréat", named after the equivalent French exam. The enrollment in first year primary school is estimated at $88.3 \%$ in 2008 , with a noticeable difference between male $(94.3 \%)$ and female $(82.3 \%)$. However, transition and survival rate are fairly low, with $16 \%$ of the total of enrolled being repeaters in the same year. Progression to secondary school is $44.4 \%$. The Baccalauréat exam concerns close to 50,000 candidates each year. The success rate went up from $40 \%$ to $50-55 \%$ during the past 5 years increasing the pressure on universities which receive relatively scarce allowances for investment in new infrastructures (see Makosso, 2006). The number of migrants with Cameroonian citizenship was less than $1 \%$ of the population. They tend to be long term migrants. In stark contrast, migration

\footnotetext{
${ }^{6}$ The data presented in this section are compounded from different reports released by: (1) international organizations, principally the 2009 Report of the International Organization for Migration (IOM) on the national profile of migration in Cameroon, the database of the UNESCO Institute for statistics, accessible online, and the World Development Indicators as released by the World Bank in 2011 and (2) the National Institute of Statistics (INSEE) of Cameroon and the Cameroonian Ministry of Education.
} 
of skilled individuals is of relatively high magnitude. The ratio of skilled migrant to the population of skilled non-migrant is $17.2 \%$ (Docquier and Marfouk (2006)). Brain drain is, for this reason, a serious concern for the Cameroonian State. In line with the migrating trend of highly educated, the ratio of Cameroonian students enrolled in a foreign country to the total number of Cameroonian students was estimated at $14.5 \%$ in 2006. On a final note, in the database collected by Docquier and Marfouk, Cameroon ranks 25th, among countries with a population higher than 4 millions, in term of rate of migration of skilled individual to OECD in 2000 (11 other sub-Saharan African countries are part of the list of the 30 highest rates). Beine, Docquier, and Rapoport (2008) finds evidence of a detrimental Brain Drain effect in Cameroon. According to their measure, the country looses $0.1 \%$ of its skilled force relative to the situation of closed economy.

4.2. Sampling methodology and corrected estimators. For the purpose of the study, a survey has been conducted on the population of Cameroonian, aged 18 or more, having completed secondary school by obtaining the Baccalauréat. A novelty of our dataset is that it comprises information on both migrants and non-migrants.

4.2.1. Sampling Design. To reach both populations (migrant and non-migrant), we used a snowball sampling procedure through an online platform. The initial sample consisted of 22 individuals (called "seeds") contacted by the researcher. The seeds where chosen on the base of geographical (country of residence) and demographical (gender, age) to include, as much as possible, all the components of the population. Each seed were asked to answer a questionnaire and to invite as much friends as possible from the population of interest. The invitee would receive an electronic mail from his host with the detail of the survey, and a unique link to access the online questionnaire. If he/she accepted to participate, he/she was required to complete the questionnaire and invite as much friends as possible in the population of interest. Recruitment is said to occur in waves and stops when invitees fail to complete the survey or invite other friends. The wave at which $i$ is invited is the number of recruiters that separates him from the initial sample. Participation in the study was restricted to a prior invitation and each invitee received a unique token which enabled us to retrace the paths of invitation. More information on the survey implementation is available in the online appendix, section 3 .

4.2.2. Estimators. We give here an idea of the methodology to correct for biases induced by nonrandom sampling and non-response of invitees. The mathematical proofs and the modification suggested to the combinatorial bootstrap procedure advised in HMQ are relegated in the online appendix, section 2 . 
Because of the particularities of sampling procedure, Horvitz-Thompson estimators are used as unbiased estimators of the true population mean. To use these estimators, we need to compute the inclusion probability for individuals in the sample. Call $q_{k i}$, the conditional probability for individual $i$ to be invited to the survey, knowing that the survey reached wave $k$. Suppose that we are at wave $k$. The set of people invited during the previous wave, are now the set of recruiters. Call this set the active set and denote it $a_{k}$. Proposition 4 in Section 2.4 of the online appendix, shows that under some simplifying assumptions, $q_{k i}$ can be decomposed into two main terms :

- The probability that an invitee $i$ agrees to participate in the study, conditional on being invited. Under the assumption that non-response occurs at random, we estimate this probability through the ratio of response when the survey reaches wave $k$.

- And the probability that individual $i$ is invited by a recruiter in the active set. To estimate this second term, we need (1) to know the probability that $i$ knows a recruiter in the active set. To do so, we fit a model of graph, the model of Erdos-Renỳi, to our sample network, in order to estimate the probability that two individuals know each other. We further need to know (2) the probability for a recruiter $j \in a_{k}$ to invite $i$, given that the two individuals share a relationship. Under the assumption that $j$ chooses his/her invitees at random among his friends, this probability is given by the ratio of the number of invitees to the number of connections of $j$ in the population, j's degree.

4.3. Descriptive statistics. The dataset consists in 402 individuals, see Tables 1 and 2 in the online appendix. We discuss some facts on the dataset and sometimes compare characteristics of migrants and other respondants, when differences are statistically significant.

Both populations are similar with regards to their age and marital status. Migrants however, appear to obtain their secondary school degree one year earlier than the others, suggesting higher number of repeaters among the non-migrants. Respondents are predominantly male. OECD countries (especially France and North America) and african countries are the favorite destinations of migrants in our sample. The survey seems to capture too few migrants to Germany compared to existing data, even after applying our correction. We however capture a sizable proportion of migrant returning to Cameroon after their studies. $19 \%$ of respondants who once migrated are now residing in Cameroon. Concerning the education, more than $40 \%$ have completed their studies. We estimate that close to a quarter of students have acquired a Master Degree or an equivalent. The most popular fields of study are Social sciences, science and engineering. We estimate that at least $42 \%$ leave before obtaining any tertiary degree in Cameroon, and more than a third pursue at least 4 years of study abroad. 
Regarding the financement, while Parents are the primary source of financing studies in Cameroon, foreign studies expenses appear to be more often shared by members of the family or left to the charge of the migrant. Parents of OECD-migrants seem to differ from the others in education and their ownership of car. More than half the respondents declare an helper to the process of migration. This helper is in the majority of cases a male, with a university degree, with close link to the family (uncle/aunt, brother/sister) and/or lives abroad.

Hereafter, we will refer to OECD countries of two types, those with high fees (US, UK, Canada, Australia and Ireland) and those with low fees. The countries are classified according to the average tuition required for students from Cameroon, as collected by the author from official documents produced by the consulates of OECD countries. As for the time span of the survey, "High tuitions" range from $\$ 8,000$ US to $\$ 30,000$ US in addition of all living costs. Countries with low tuitions, such as Germany and France, require from $\$ 0$ US to $\$ 2000$ US each year. Australia and Ireland, which are high tuition countries, do not appear in our sample.

\section{EMPIRICAL RESUlTS}

We present first the results for the preestimation of the family capital. We then turn in Subsection 5.2 to the results of our inference procedure for the discrete choice model of student migration investment.

5.1. First-step estimation. As detailed in Section 3.4, we run a probit regression to retrieve a $95 \%$ confidence interval on the proportion of capital that a family needs to borrow in order to meet the liquidity constraint. The dependent variable is only discretely observed. In the questionnaire, respondents identify who is, will be or would have been responsible for the different costs in the event of a migration. These costs are divided in living costs, tuition costs and travel costs. The possible payers are the candidate himself, the parents, a definite helper, the government or a scholarship, or a mix of all these options. If individuals are paying, the respondents is further asked whether the costs are paid through savings, regular income, borrowing from an individual or from an institution, a mix of the previous options or from other means. We will consider that the budget constraint is binding, if one of the payer provides the funds through the means of a loan, or if a non-migrant expects the government to pay the fees for the migration.

For a first regression, including the whole sample, results are counter-intuitive, as the capital of the family is unrelated to its physical capital (ownership of a car or a house) and negatively correlated to the parents education. This suggests that non-migrants differ significantly from migrants in their evaluation of the costs. As they do not have full information on migration costs, non-migrants 
appear to provide unreliable estimates of the ability of the family to meet the costs of the migration. We therefore only use the subsample of 132 migrants to the OECD. Results are displayed in Table 1 for several specifications.

The results show that obtention of a scholarship significantly decreases the liquidity constraints (Scholarships available for students in Cameroon are mainly on the basis of merit and not on the basis of need). Number of cars owned by the family (the parents and helper when present) as well as maximal family education are also good predictors of the family capital. This latter result concords with the findings of National Institute of Statistics of Cameroon, in their survey on Cameroonian Household in 2006.

What do we learn about the helper? Interestingly, once we have controlled for the physical capital and the education of a helper, that he/she resides in the country of migration does not appear as a significant source of capital for the family. Our data do not support the idea that a migrant will hold significant implicit capital from the location of a low-educated helper with low capital in the host country. The measure of the support provided even decreases in absolute value when we control for the number of siblings abroad.

5.2. Inference on parameters of the structural model of Investment. We conduct inference on the model described in section 2 with two levels of education: $E d=1$ if the individual has at least a Masters Degree, $E d=0$ otherwise.

5.2.1. Model specification. We use the following specification of the return of individual $i$ for choosing alternative $j, j \in\{0,1\}$ in eq. (2.2)

$$
\begin{aligned}
& \tilde{\pi}_{0}=0 \\
& \tilde{\pi}_{1}=\mu+\alpha \cdot E d_{i}+X_{i} \cdot \beta^{\prime}+r_{0} \cdot \sigma_{u} \cdot \min \left(\hat{\bar{V}}_{1}\left(L_{i}\right), 0\right)
\end{aligned}
$$

where:

- $\hat{\bar{V}}\left(L_{i}\right)$ denotes the estimate of the proportion of the investment that a family needs to borrow. We use Specification 2 in Table 1.

- $X_{i}$ are the characteristics of individuals which might influence the success of the migration.

- Again, $\varepsilon$ is assumed to have a logistic distribution with normalized variance (equal to 1).

In this specification, $\alpha$ measures the additional return from holding a Master degree in a foreign country. The characteristics of the individuals that will be of interest are the Gender (with parameter $\left.\beta_{G}\right)$, the primogeniture $\left(\beta_{\text {prim }}\right)$, the country of residence of the helper $\left(\beta_{\text {resid }}\right)$ and the success of the child in secondary education $\left(\beta_{\text {qual }}\right)$. To measure the latter, we construct a dummy variable 
TABLE 1. Probit regression of the Proportion of capital that a family must borrow, conditional on emigration to OECD countries.

\begin{tabular}{lrrrr}
\hline \hline & Spec 1 & Spec 2 & Spec 3 & Spec 4 \\
\hline Intercept & $-0.564^{* *}$ & $1.187^{* * *}$ & $1.225^{* * *}$ & $1.313^{* * *}$ \\
& $(0.326)$ & $(0.415)$ & $(0.432)$ & $(0.444)$ \\
Grant & $-1.112^{* * *}$ & $-1.927^{* * *}$ & $-2.024^{* * *}$ & $-2.050^{* * *}$ \\
& $(0.410)$ & $(0.517)$ & $(0.515)$ & $(0.487)$ \\
OECD High Tuition & $0.934^{* *}$ & $0.849^{* *}$ & $0.745^{* *}$ & 0.226 \\
& $(0.396)$ & $(0.370)$ & $(0.363)$ & $(0.394)$ \\
\hline Family characteristics & & & & \\
\hline Maximum parent education & & $-0.370^{* * *}$ & $-0.312 * *$ & $-0.291 * *$ \\
& & $(0.132)$ & $(0.137)$ & $(0.142)$ \\
Number of cars owned & & $-0.481 * *$ & $-0.440 * *$ & $-0.417 * *$ \\
Helper in Migration Country & & $(0.202)$ & $(0.194)$ & $(0.172)$ \\
OCDE high $\times$ Nbr children abroad & & & -0.469 & -0.165 \\
& & & $(0.355)$ & $(0.350)$ \\
OCDE low $\times$ Nbr children abroad & & & & 0.364 \\
log likelihood & & & & $-0.291)$ \\
AIC & -34.983 & -27.340 & -26.798 & -25.338 \\
\hline \hline
\end{tabular}

Number of obs. $=132$. Estimation is made for a normal standard error term. Standard deviation are in parentheses. (***) significant at $1 \%$. (**) significant at $5 \% .\left({ }^{*}\right)$ significant at $10 \%$.

which is one if the candidate to migration has succeeded the Baccalauréat with honors. We interact this dummy variable with another variable relative to the age at which the candidate passed the Baccalauréat. This variable is zero if the individual was older than the median person, and equal to the difference of age if younger ${ }^{7}$. A positive coefficient suggest positive selection of the migrant.

\footnotetext{
${ }^{7}$ The educational system displays a high rate of repeaters at every level. According to UNESCO statistics, around $20 \%$ in primary school and $14 \%$ in each year of Secondary school. Younger students
} 
Regarding enrollment in first year primary school, families in Cameroon exhibit a strong preference for male children over female children. It is of much interest to understand whether this preference survives through the process of student migration. The literature on migration of high skilled individuals suggests that such gender gap may not exist (see survey from Docquier and Rapoport (2009)). Related to the fact that helpers are most commonly brothers of the candidate, we expect firstborn children to suffer from a lack of support. A complementary explanation might be provided by the literature on elderly parents care. Firstborn children are often chosen to provide care for elderly parents and distance deters the readiness of the child to provide such care (see for example Engers and Stern (2002), Rainer and Siedler (2009)). Since student migration might ultimately result in permanent migration, family members (mostly parents) might be reluctant to send the eldest child abroad. Residence of the helper in the migration country was found not to add to the capital of the family once we control for the education and physical capital of the helper. We explore whether it influences in alternative ways the return of migration. We think here of some non-pecuniary return of having a relative living in the same country. The literature on economic migration acknowledges the existence of psychological cost that may arise from separation from one's native that might be reduced by the presence of a previous migrant in the host country (Bauer, Epstein, and Gang (2000), Mahmood and Schömann (2003)). We will also explore whether the fact that parents have a past experience of migration $\left(\beta_{\exp }\right)$ influences the migration probability. A number of studies (for example BLV) use variables related to the past experience of migration of parents as exclusion restriction in the equation of migration ${ }^{8}$.

Significance of the individual parameters is evaluated by checking whether the hyperplanes defined by $\theta_{i}=0$ - where $\theta_{i}$ is a component of $\theta$ - intersect the $90 \%$ confidence region. We report the range for each parameters in Table 2 .

5.2.2. Discussion of results. An important result is that the differential in the return from holding a Masters degree has a strong positive effect on incentive to study abroad $(\alpha>0)$. To give and idea of the magnitude of $\alpha$, we can interpret the variation of the odd ratio defined as the probability to migrate relative to the probability not to migrate. Our finding implies that, controlling for other covariates, the odds of migration will at least double when individual choose to study at least to Master Degree. Although families exhibit a clear preference for studies in Cameroon rather than

are then expected to have finished primary and secondary school without repeating a class, and to be better than the average.

${ }^{8} \mathrm{We}$ also used in an alternative specification the past experience of student migration of the parent. This variable does not appear to be significant. 
abroad $(\mu<0)$, their disutility is entirely compensated by the return from holding a foreign Master degree.

TABle 2. Parameter ranges

\begin{tabular}{lrr}
\hline \hline & Min & Max \\
\hline Master in foreign Country $(\alpha)$ & 0.88 & 3.61 \\
Cst. $(\mu)$ & -0.38 & -0.13 \\
Female $\left(\beta_{G}\right)$ & -0.90 & 0.10 \\
Primogeniture $\left(\beta_{\text {prim }}\right)$ & -0.70 & -0.29 \\
Academic results $\left(\beta_{\text {qual }}\right)$ & 0 & 2.41 \\
Migration experience $\left(\beta_{\text {exp }}\right)$ & -0.50 & 0.25 \\
Residence of helper $\left(\beta_{\text {resid }}\right)$ & -0.41 & 0.04 \\
\hline \hline
\end{tabular}

$r_{0} \sigma_{u}$ is not reported since uninformative.

Our measure of the success of the child during secondary school appears positively correlated with the chances of migration. We reject $\beta_{\text {qual }}<0$, which means that the best students are more likely to migrate. This suggests a positive selection of migrants. An interesting result is that the firstborn child is less likely to migrate than his younger siblings. Indeed, his/her odds of migration are $25 \%$ smaller. This result is not surprising as a third of the helper reported are (older) brothers or sisters. Investigation of chain migration within families might be of great interest. We find no overwhelming evidence that the male students are favored by the family in the migration process, (we cannot rule out $\beta_{G}=0$ ), a result in line with the main findings in the previous literature on migration. Nevertheless, the confidence region shows a tilt toward negative values of this parameter. Our data are not compatible with models were strong preference is given to migration of female students. When we study instead a dummy variable for the presence of the helper in the migration country (not reported), the range of $\beta_{\text {resid }}$, includes 0 (and is almost symmetric around this value). In the same line, when we consider instead residence of the helper in Cameroon, we cannot rule out $\beta_{\text {resid }}=0$, and models where there exists a positive effect from having an helper residing in Cameroon are almost always rejected. Whether the helper is from diaspora or living in Cameroon, does not seem to be a significant variable for the final outcome. The role of the helper seems then to be limited to provision of (explicit or implicit) capital to the migration process. To reconcile this result with the findings of the literature about the network effect of the diaspora, we must conlude that the lessening of psychological costs is then not related to a sole individual but to a larger network that the migrant can build. Finally, the past migration experience of the parents does not 
appear as a significant determinant of the migration of the child, a finding warranting caution in the use of this variable as exclusion restriction.

\section{Conclusion}

This paper presented a framework to analyze the determinants of the choice of migration from a microeconomic perspective. We do so while overcoming the main obstacles to a rigorous study, namely: the lack of data about population of migrants and non-migrants and the technical difficulty raised by the endogeneity of the educational attainment in our discrete-choice model. We deal with the first of these issues using a novel chain-referral sampling procedure, run through an online platform. The procedure allowed to overcome the geographical challenge posed by the type of population of interest. We proposed population mean estimators that correct for biases induced by non-response and non-random sampling. In practice, strong assumptions on recruitment behaviors are needed to retrieve the inclusion probabilities of sampled individuals. We also need to fit a random graph model to represent the relation pattern between individuals in the population. The choices made in this paper were for computational reason.

We propose an empirical structural decision model which reflects the importance of both the return of the investment and the budget constraint in agent choices. An important contribution of this paper is the appeal to recent results in the literature about incomplete, partially identified models to circumvent the problem of endogeneity. In doing so, we relax the point identification condition for the parameters of our structural model of investment in student migration. We conduct inference using sharp bounds and retrieve confidence regions which appear quite informative. Another contribution of the paper is the novel two-steps inference methodology that takes into account censored or incompletely observed variables and considerably reduce the computational burden.

From our sample, we find that a higher educational level and better results during secondary education of the candidate increase significantly the returns to migration. We find also that male students are not favored by the family in the migration process and the firstborn child is less likely to migrate than his younger siblings. Our interpretation is that they suffer from lack support from an elder brother/sister. Our survey data are actually quite informative about the helper who appears to be in the majority of the cases a male relative of the parent and the child, with tertiary education. When present, he influences primarily the budget constraint of the family.

\section{REFERENCES}

Batista, C., A. Lacuesta, and P. C. Vicente (2012): "Testing the 'brain gain' hypothesis: Micro evidence from Cape Verde," Journal of Development Economics, 97(1), 32-45. 
Bauer, T. K., G. Epstein, AND I. Gang (2000): "What are migration networks?," IZA Discussion paper.

Beine, M., F. Docquier, And H. Rapoport (2008): "Brain drain and human capital formation in developing countries: winners and losers," Economic Journal, 118(528), 631-652.

Beine, M., R. Noel, And L. Ragot (2012): "The determinants of international mobility of students," CESifo Working Paper: Economics of Education, No. 3848.

Beresteanu, A., I. Molchanov, And F. Molinari (2011): "Sharp identification regions in models with convex predictions," Econometrica, 79, 1785-1821.

Bessey, D. (2011): "International student migration to Germany," Empirical Economics, 42(1), $345-361$.

BorJas, G. J. (1989): "Economic Theory and International Migration," International Migration Review, 23(3), pp. 457-485.

Borjas, G. J., AND B. Bratsberg (1994): "Who Leaves? The Outmigration of the ForeignBorn," SSRN eLibrary.

Brezis, E., AND A. Soueri (2011): "Why students migrate? Where do they migrate to?," Working Papers 25, AlmaLaurea Inter-University Consortium.

Chernozhukov, V., H. Hong, And E. Tamer (2007): "Estimation and Confidence Regions for Parameter Sets in Econometric Models," Econometrica, 75, 1243-1285.

Chernozhukov, V., S. Lee, And A. Rosen (2009): "Inference on intersection bounds," CEMMAP Working Paper CWP19/09.

Chesher, A., A. Rosen, And K. Smolinski (2011): "An instrumental variable model of multiple discrete choice," CEMMAP Working Paper CWP06/11.

Ciliberto, F., ANd E. TAmer (2009): "Market structure and multiple equilibria in airline markets," Econometrica, 70, 1791-1828.

Docquier, F., AND A. MARFouk (2006): "International migration by educational attainment, 1990 - 2000," Caglar Ozden, Maurice Schiff, Editors, International Migration, Remittances, and the Brain Drain.

Docquier, F., AND H. RAPOPORT (2009): "The economics of brain drain," unpublished manuscript.

Dreher, A., AND P. PoutvaAra (2011): "Foreign students and migration to the United States," World Development, 39(8), 1294-1307.

Engers, M., AND S. Stern (2002): "Long-term care and family bargaining," International Economic Review, 43(1), 73-114.

Galichon, A., AND M. Henry (2006): "Dilation Bootstrap," unpublished manuscript. 
(2011): "Set identification in models with multiple equilibria," Review of Economic Studies, $78,1264-1298$.

Hanson, G. H., And C. Woodruff (2003): "Emigration and educational attainment in Mexico," Mimeo.

Heckathorn, D. D. (1997): "Respondent-Driven Sampling: A New Approach to the Study of Hidden Populations," Social Problems, 44(2), pp. 174-199.

(2007): "Extensions of Respondent-Driven Sampling: Analyzing Continuous Variables and Controlling for Differential Recruitment," Sociological Methodology, 37, pp. 151-208.

Henry, M., R. MÉAngo, And M. Queyranne (2011): "Combinatorial bootstrap inference in partially identified incomplete structural models," unpublished manuscript.

Jovanovic, B. (1989): "Observable implications of models with multiple equilibria," Econometrica, 57, 1431-1437.

Mahmood, T., And K. Schömann (2003): "On the Migration Decision of IT-Graduates: A Two-Level Nested Logit Model," WZB Markets and Political Economy Working Paper No. SP II, 22 .

MAllaR, C. D. (1977): "The estimation of simultaneous probability models," Econometrica: Journal of the Econometric Society, pp. 1717-1722.

MANSKI, C. (1993): "Identification of endogenous social effects: the reflection problem," Review of Economic Studies, 60, 531-542.

McKenzie, D., AND H. RAPOPORT (2007): "Network effects and the dynamics of migration and inequality: Theory and evidence from Mexico," Journal of Development Economics, 84(1), 1 - 24. Nemb, P. S., And U. E. Jumbo (2011): "Banks and tontines: Complementarity or Competition? The case of Cameroon," International Finance and Business Journal, 1(1), 30-40.

Perkins, R., And E. Neumayer (2011): "Educational mobilities in an age of internationalization: quality, social ties and border controls in the uneven flows of foreign students," in EUGEO session of the Royal Geographical Society Annual International Conference, London, vol. 31.

RAIneR, H., AND T. Siedler (2009): "O brother, where art thou? The effects of having a sibling on geographic mobility and labour market outcomes," Economica, 76(303), 528-556.

Rosenzweig, M. R. (2008): "Higher Education and International Migration in Asia: Brain Circulation," in Annual World Bank Conference on Development Economics, pp. 59-100.

Rosenzweig, M. R., D. A. Irwin, And J. G. Williamson (2006): "Global Wage Differences and International Student Flows [with Comments and Discussion]," in Brookings Trade Forum, pp. 57-96. JSTOR.

Spence, M. (1973): “Job market signaling," The Quarterly Journal of Economics, 87(3), 355-374. 
Thissen, L., AND S. Ederveen (2006): Higher education: Time for coordination on a European level?, no. 68. CPB Netherlands Bureau for Economic Policy Analysis.

Thompson, S. K. (2006): "Adaptive Web Sampling," Biometrics, 62(4), 1224-1234.

Van Bouwel, L., AND R. Veugelers (2009): "The determinants of student mobility in Europe: the quality dimension," FBE Research Report MSI_0912, pp. 1-39.

Wejnert, C., And D. D. Heckathorn (2008): "Web-based network sampling: Efficiency and efficancy of Respondent-Driven Sampling for online research," Sociological Methods and Research. WiLlis, R. J. (1985): Wage determinants: A survey and reinterpretation of human capital earnings functions. Economics Research Center/NORC. 